\title{
LA HORTICULTURA EN LA ZONA CENTRAL DE CHILE: CARACTERIZACIÓN Y ACTITUDES DE LOS PEQUEÑOS AGRICULTORES
}

\section{VEGETABLE GROWING IN CENTRAL CHILE: CHARACTERIZATION AND ATTITUDES OF SMALL FARMERS}

\author{
Sofía Boza ${ }^{1 *}$, Maruja Cortés ${ }^{1}$, Carmen Prieto ${ }^{2}$, y Tomás Muñoz ${ }^{1}$ \\ ${ }^{1}$ Departamento de Economía Agraria y Centro Cultiva UChile, Facultad de Ciencias Agronómicas, \\ Universidad de Chile. Santa Rosa 11315, La Pintana, Santiago, Chile. \\ 2 Departamento de Agroindustria y Enología y Centro Cultiva UChile, Facultad de Ciencias Agronómi- \\ cas, Universidad de Chile. Santa Rosa 11315, La Pintana, Santiago, Chile. \\ * Autora para correspondencia E-mail: sofiaboza@u.uchile.cl
}

\section{RESUMEN}

La oferta de hortalizas frescas, de calidad y accesibles, es esencial para mejorar la dieta en Chile. La zona central es la principal área productiva del país. El objetivo de la presente investigación fue caracterizar a los pequeños productores hortícolas de dicha zona, y determinar sus actitudes respecto a su actividad. Para ello se encuestó de forma presencial a un total de 165 agricultores en 10 comunas de la Región de O'Higgins. Los datos obtenidos fueron tratados por medio de técnicas descriptivas. Entre los resultados alcanzados destacan los siguientes: i) el promedio de edad es de 52,47 años, ii) el 53,34\% de los encuestados no tiene educación formal o ha finalizado sólo la básica, iii) hay un buen acceso a servicios y movilización, iv) el promedio de hectáreas productivas es 7,8 , pero con alta dispersión, v) es muy limitado el uso de invernadero, técnicas de agricultura orgánica e hidroponía, y la contratación de mano de obra permanente, vi) el uso de Tecnologías de Información y Comunicaciones (TIC) - excepto celular - es escaso, vii) la innovación declarada se centra en aspectos técnico-productivos, viii) la mayor parte de los encuestados no se asocia, su relación con el sector público es acotada, vende a través de intermediarios y no lleva registro de sus operaciones. Respecto a sus actitudes, destaca la valoración positiva de la manera en la cual realizan su actividad productiva; sin embargo, existe incertidumbre sobre el futuro de la misma.

Palabras clave: Horticultura, hortalizas, pequeño agricultor, pequeña agricultura, Región de O'Higgins.

\section{ABSTRACT}

The supply of fresh, high-quality and easily accessible vegetables is essential to improve the diet in Chile. The central zone is the main production area of the country. The objective of this research was to characterize small vegetable growers of the central zone, as well as to determine their attitudes towards their activity. For this, a total of $\mathbf{1 6 5}$ farmers distributed in $\mathbf{1 0}$ counties of the O'Higgins Region were surveyed in person. The data obtained were treated by descriptive techniques. The principal results are the following: $i$ ) the average age of the farmers surveyed is 52.47 years, $\mathbf{i i}) 53.34 \%$ of them have no formal education or have completed only primary education, iii) there is good access to services and transportation, iv) the productive hectares average is 7.8 , but with high dispersion, v) the use of greenhouses, organic farming and hydroponics is very limited, while most workers are not hired on permanent basis, vi) the use of Information and Communication Technologies (ICT) except for cell phones- is scarce, vii) innovation focuses on technical-productive aspects, viii) most of

Recibido: 12 septiembre 2018. Aceptado: 28 enero 2019. 
the respondents do not associate with other farmers and, their relationship with the public sector is limited; they sell their products through intermediaries and do not keep records of their operations. Regarding their attitudes, the surveyed farmers make a positive evaluation of their work. However, they experience a sense of uncertainty about the future.

Key words: Vegetable growing, vegetables, small farmer, small-scale farming, O’Higgins Region.

\section{INTRODUCCIÓN}

Desde hace varias décadas el sector agrícola chileno ha seguido una senda de intensificación de su orientación exportadora, posicionándose como referente en el Hemisferio Sur. No obstante, la inserción comercial ha sido asimétrica, dependiendo del rubro. El año 2017 el valor de las exportaciones chilenas de frutas frescas fue de 4.800 millones de dólares, mientras que las hortalizas frescas sumaron solamente 41,9 millones de dólares (ODEPA, 2018).

La escasa inclusión en los mercados internacionales ha derivado en menor desarrollo de la horticultura en términos generales. Echeverría et al. (2012) encontraron que en Chile los agricultores orientados al mercado nacional tienen un notorio atraso en uso de tecnologías, acceso a financiamiento e incluso a programas públicos, comparados con aquellos focalizados en la exportación. En este sentido, Ríos y Torres (2014) afirman que la agricultura chilena se caracteriza por una marcada dualidad entre una minoría de empresas medianas y grandes orientadas a la exportación, y una mayoría de micro y pequeños productores orientados al mercado local, lo que conlleva significativos contrastes en las realidades presentes.

Efectivamente, como en otros cultivos intensivos en mano de obra, en la horticultura nacional tiene una participación muy relevante la pequeña agricultura (Berdegué y Rojas, 2014). A nivel mundial se estima que este tipo de agricultura acumula un $98 \%$ de los predios y un 53\% de las tierras (Graeub et al., 2016), pero se encuentra en un proceso de abandono hacia otras actividades que se perciben como más viables económicamente (Van Vliet et al., 2015). En Chile según cifras del Banco Mundial (2018) la población rural ha decrecido en un $0,8 \%$ anual en las últimas dos décadas, mientras que la urbana ha aumentado un $1,5 \%$.

Sin embargo, pese a las limitaciones, la pequeña agricultura chilena es un proveedor fundamental de frutas y hortalizas frescas, sobre todo a través de las ferias libres (Tejada, 2013), donde son el principal producto comercializado (Observatorio Feria Libre, 2013).

En Chile más de un tercio de la población es obesa, casi el $40 \%$ tiene sobrepeso y sólo el $5 \%$ se alimenta de forma saludable (Universidad de Chile, 2014; Ministerio de Salud, 2017a). En ese escenario, disponer de productos frescos, de calidad y asequibles es crucial. De hecho, las recomendaciones al público general de los especialistas en nutrición y de las instituciones chilenas, coincidentes en la importancia de promover el consumo de hortalizas, son una relevante oportunidad para los productores. En este sentido, el Ministerio de Salud (2017b) declara su propósito de trabajar en las políticas públicas de alimentación y nutrición desde un enfoque de "entornos alimentarios". Éstos son espacios complejos donde interaccionan una alta multiplicidad de factores que condicionan las decisiones de consumo de los individuos (Cerda et al., 2016). En el caso del acceso de los ciudadanos a hortalizas frescas en Chile, se evidencia que está efectivamente determinado por el entorno alimentario, el cual incide en la disponibilidad (Espacio y Fomento, 2016).

La zona central de Chile concentra la mayor parte de la población del país, y en consecuencia de la demanda de productos frescos. Así mismo, según cifras de la Encuesta de Superficie Hortícola del Instituto Nacional de Estadísticas (INE, 2016), el $75 \%$ de la superficie dedicada a las hortalizas en Chile se encuentra en la zona central. La Región del Libertador Bernardo O'Higgins (en adelante Región de O'Higgins) es la segunda a nivel nacional en número de hectáreas hortícolas tras la Región Metropolitana de Santiago.

El objetivo de la presente investigación fue caracterizar a los agentes que constituyen el eslabón inicial de la oferta de hortalizas en Chile, esto es, a los productores, tomando para ello el caso de la zona central del país y, en específico, de la Región de O'Higgins. Así mismo, se busca conocer las opiniones personales de los horticultores respecto de su actividad. Consideramos que este esfuerzo constituye un aporte para el diseño de políticas públicas que contribuyan al fomento del rubro y a la mejora de la seguridad alimentaria y nutricional nacional, ya que facilita información actualizada desde un enfoque escasamente explorado.

\section{MATERIALES Y MÉTODOS}

La información analizada en el presente artículo se obtuvo a partir de la realización de 165 encuestas a productores hortícolas ubicados 
en la Región de O'Higgins (coordenadas capital regional, Rancagua: $34^{\circ} 09^{\prime} 55^{\prime \prime}$ S $70^{\circ} 44^{\prime} 23^{\prime \prime} \mathrm{O}$ ). Los agricultores encuestados se distribuían en las comunas de: Chépica, Coltauco, Las Cabras, Malloa, Peumo, Pichidegua, Quinta de Tilcoco, Rengo, SanFernandoySan VicentedeTaguaTagua. La muestra total fue definida por conveniencia, así como la identificación de los individuos a entrevistar. Es decir, la muestra no fue aleatoria, sino que se fijó el número de encuestas que era posible considerando los medios disponibles y la selección de los encuestados se realizó una vez en terreno teniendo en cuenta aquellos agricultores que formaran parte de la población objetivo a los que se tuvo acceso. Si bien esto reduce la representatividad de la información obtenida respecto a la población bajo estudio - pequeños horticultores de la Región de O'Higgins - se trata de un muestreo adecuado para la realización de un análisis exploratorio de la realidad (Etikan et al., 2016).

Las encuestas fueron realizadas de manera presencial durante los meses de enero y febrero de 2017. Con posterioridad se llevó a cabo un proceso de validación y codificación de la información levantada. Las preguntas que se consideraron en la encuesta se dividieron en las siguientes categorías: i) identificación de los encuestados, ii) perfil socio-demográfico, iii) caracterización técnico-productiva de la explotación hortícola, iv) acceso a Tecnologías de Información y Comunicaciones (TIC), realización de innovaciones y uso de sistemas de aseguramiento de la calidad e inocuidad, v) mecanismos de gestión predial y de comercialización de la producción, y vi) actitudes acerca de su actividad.

En el cuestionario se utilizaron preguntas cerradas de elección única - tanto dicotómica como politómica - para los ítems del i) al v). Para la última categoría se recurrió a una escala Likert (Santesmases, 2009) de cinco niveles; en este caso, 1: "totalmente en desacuerdo", 2: "en desacuerdo", 3: "indiferente", 4: "de acuerdo", y 5: "totalmente de acuerdo". La información obtenida fue tratada mediante técnicas de análisis descriptivo, específicamente frecuencias y medidas de tendencia central (promedios) en el caso de las preguntas cerradas, sumado a análisis de dispersión (desviación estándar) para las respuestas referentes a actitudes.

\section{RESULTADOS Y DISCUSIÓN}

\section{Antecedentes generales de los encuestados}

En primer lugar, se consultó a los encuestados acerca de su perfil socio-demográfico: género, edad y nivel educacional más alto completado. Los principales resultados obtenidos pueden observarse en la Tabla 1.

Tabla 1. Antecedentes generales de los horticultores encuestados, Región de O'Higgins, 2017. Table 1. General information of the vegetable growers surveyed, O'Higgins Region, 2017.

\begin{tabular}{lcc}
\hline Categoría & Frecuencia & Porcentaje \\
\hline Género & & \\
$\quad$ Hombre & 151 & 91,1 \\
$\quad$ Mujer & 14 & 8,49 \\
Edad & & \\
$\quad$ Entre 20 y 29 años & 5 & 3,03 \\
Entre 30 y 39 años & 18 & 10,91 \\
Entre 40 y 49 años & 43 & 26,06 \\
Entre 50 y 59 años & 55 & 33,33 \\
Entre 60 y 69 años & 26 & 15,76 \\
Más de 70 años & 17 & 10,30 \\
N.C. & 1 & 0,61 \\
Nivel de educación & & \\
Ninguno & 21 & 12,73 \\
Básico & 67 & 40,61 \\
Secundaria & 53 & 32,12 \\
Técnica & 10 & 6,06 \\
Superior & 11 & 6,67 \\
N.C. & 3 & 1,82 \\
\hline
\end{tabular}

N.C.: No contesta. 
De los 165 encuestados 151 fueron hombres y 14 mujeres. Esta diferencia puede deberse a que el muestreo fue por conveniencia, por lo que no existieron criterios previos de selección. No obstante, contrasta con la cifra de $30 \%$ de jefatura predial femenina en Chile $(80.000$ mujeres) conforme al Censo Agropecuario de 2007 (Qualitas, 2010). De hecho, la creciente presencia de mujeres como jefas de explotación es un fenómeno que se observa a nivel mundial, especialmente en la pequeña agricultura (FAO, 2011; NEPAD, 2013; Wilchens, 2015). En todo caso, su constante movilidad entre distintas actividades remuneradas y no remuneradas hace que la participación de la mujer en el sector agrícola chileno sea difícil de cuantificar, lo que lleva a tener cifras que difieren según la fuente consultada (Namdar-Irani y Aracena, 2014).

El promedio de edad de los encuestados fue de 52,47 años. Solamente el 13,94\% estaba por debajo de los 39 años. Estos resultados son coincidentes con investigaciones previas que evidencian que la edad promedio de los pequeños agricultores en Chile está por lo general en el intervalo de los 50 a los 55 años (Vera y Moreira, 2009; Boza et al., 2015; 2016). Además, en la la zona central de Chile se observa una sostenida migración rural-urbana, la cual es protagonizada principalmente por los más jóvenes (Castro, 2012; Valdés y Rebolledo, 2015), lo que incide en el envejecimiento poblacional de las zonas estudiadas.

Un 53,34\% de los encuestados no ha completado ningún nivel de educación formal o tiene solamente educación básica. A nivel nacional, conforme a la encuesta CASEN 2017, el 24,6\% de la población mayor de 18 años se encontraba en la misma situación, aunque la cifra es del 49,2\% considerando solamente los habitantes de áreas rurales. El porcentaje de personas con educación superior es también notoriamente inferior al promedio nacional, del 19,8\%.

\section{Perfil socio-demográfico}

En la segunda parte de la encuesta se consultó a los productores acerca de las características básicas de su hogar, del territorio donde habitan y de su vivienda, la composición familiar, el acceso a servicios y los medios de movilización utilizados.

El promedio de personas que compone el hogar de los encuestados es de 2,25. Más de la mitad de los productores tienen un hogar conformado por 2 o 3 miembros adicionales, y solamente un $18,79 \%$ tiene 4 o más, además del propio productor. La merma del número de integrantes en el hogar es un fenómeno que se ha acentuado en Chile en los últimos años. En el Censo de 2002 se registraban hogares con un promedio de 3,57 miembros, en 2012 con 3,28 y en 2017 con 3,1. La menor natalidad y la migración hacia las ciudades explican esta evolución. La inmigración se prevé como una alternativa para contrarrestar, al menos en parte, el envejecimiento poblacional actual y sobre todo futuro (Bravo y Urzúa, 2018). En los productores encuestados, en un $60,6 \%$ de los casos ningún miembro del hogar era un menor. Un $72,7 \%$ de los encuestados vive en pareja. En un $36,9 \%$ de los casos, algún otro miembro del grupo familiar trabaja en el predio. En este punto, cabe mencionar que una característica que de forma consensuada se le atribuye a la pequeña agricultura, es la estrecha vinculación entre predio y hogar (Garner y Campos, 2014).

Un 96,4\% de losencuestados afirmótener acceso a servicios de salud, principalmente consultorio y posta rural. Por su parte, un $98,2 \%$ dijo tener acceso en su zona a servicios educacionales. El nivel educacional que los productores declararon como disponible fue: básico $(37,7 \%)$, secundario $(8 \%)$, técnico $(45 \%)$ y universitario $(9,3 \%)$. La falta de espacios para la formación superior, sobre todo en carreras tecnológicas, es un problema en la Región de O'Higgins. Esto conlleva que los jóvenes estudien en otras regiones; pérdida de futuro capital humano que a posteriori es difícil de revertir (Gobierno Regional de O'Higgins, 2012). Casi la totalidad de los encuestados señaló tener acceso a agua potable en su vivienda $(98,2 \%)$, pero sólo un $12,1 \%$ a la red de alcantarillado. Un $98,2 \%$ de los encuestados tiene electricidad en su vivienda, así como telefonía fija; pero sólo un $24,2 \%$ tiene acceso a Internet.

En relación a la movilización, un $94,5 \%$ de los encuestados afirmó utilizar principalmente locomoción particular. Un $81,2 \%$ de los productores considera que el estado de la red vial que comúnmente utiliza es "muy bueno" o "excelente". Estos resultados son muy relevantes, dado que la autonomía para movilizarse facilita a los productores el acceso a mercados tanto para vender productos como para la compra de insumos, al igual que la realización de trámites.

\section{Caracterización técnico-productiva de la explotación}

Los productores fueron consultados respecto al perfil técnico-productivo de sus explotaciones, en aspectos como: tamaño, régimen de tenencia, especies hortícolas cultivadas, modos de producción, ingresos por venta, y mano de obra requerida.

El tamaño promedio total del predio de los encuestados era de 10,31 hectáreas, mientras que el área productiva era en promedio de 7,8. Sin embargo, un $38,8 \%$ de los encuestados manejan predios con menos de 5 hectáreas totales 
y un $41,2 \%$ de los encuestados con menos de 5 hectáreas productivas. En todo caso, la superficie operada por los agricultores consultados es notablemente mayor que el promedio para los productores de hortalizas en la región, de 2,08 hectáreas según datos del Censo Agropecuario (INE, 2007). Un 69,1\% de los encuestados es arrendatario de las tierras que maneja, mientras que el $26,6 \%$ es propietario.

Los cultivos con mayor superficie entre los productores encuestados son cebolla (Allium сера), papa (Solanum tuberosum), ajo (Allium sativum), melón (Cucumis melo) y tomate (Solanum lycopersicum); lo que en términos generales es coincidente con las cifras regionales reportadas por el INE (2016), aunque en ese caso el zapallo (Curcubita maxima) y el choclo (Zea mays) tienen mayor presencia. El $95,2 \%$ de los productores cultivan al aire libre; el uso de invernaderos es por tanto muy limitado (4,8\%). El 89,7\% de los encuestados utiliza riego por surco. El 98,2\% de los agricultores usa técnicas convencionales; la hidroponía y el cultivo orgánico son muy marginales $(1,8 \%)$. En todo caso, la presencia de la agricultura orgánica en la horticultura nacional es en general baja; el total registrado en 2016 era de sólo 500 hectáreas (Eguillor, 2017).

El ingreso familiar promedio mensual declarado por los productores fue de $\$ 2.047 .334$ (1 dólar $=\$ 695,6 ; 12-09-2018)$ y $\$ 844.365$ el de la explotación, aunque con una notoria dispersión. Un $65,45 \%$ de los horticultores señala que no trabaja fuera del predio. Estos resultados sugieren que probablemente otros miembros del hogar están aportando al ingreso familiar. Respecto al número de trabajadores que tienen contratados en la explotación de forma permanente, el promedio es de 0,33. De hecho, un $81,21 \%$ de los encuestados no tenía ningún trabajador permanente contratado. Estos resultados concuerdan con que, como señala Maloney (2004), en las labores agrícolas priman las relaciones de índole temporal, así como los contratos de palabra basados en la confianza dado que no se entiende la necesidad de formalizarlos.

\section{Acceso a TIC, innovación y certificaciones de los encuestados}

Adicionalmente, se consultó a los encuestados acerca de la penetración de las TIC en sus negocios, las innovaciones realizadas recientemente y el uso de sistemas de aseguramiento de la calidad e inocuidad. Los principales resultados se pueden observar en la Tabla 2.

En primer lugar, llama la atención el uso del computador de solamente el $30,3 \%$, en contraste con la telefonía celular, del $96,36 \%$. En efecto, la penetración del computador y de Internet son minoritarias en los pequeños agricultores, en especial en las explotaciones de reducido tamaño

Tabla 2. Uso de TIC, innovación y certificaciones de los encuestados.

Table 2. Use of ICT, innovation and certifications of the respondents.

\begin{tabular}{lrrrc}
\hline Categoría & Sí & \% & No & $\%$ \\
\hline Uso de celular & 159 & 96,36 & 6 & 3,64 \\
Uso de computador & 50 & 30,3 & 115 & 69,7 \\
Uso de correo electrónico & 26 & 15,76 & 139 & 84,24 \\
Realización de trámites online & 21 & 12,73 & 144 & 87,27 \\
Innovación en los últimos 5 años en: & 120 & 72,72 & 45 & 27,28 \\
Especies & 106 & 64,24 & 59 & 35,76 \\
Manejos de campo & 23 & 13,94 & 142 & 86,06 \\
Prácticas de gestión & 56 & 33,94 & 109 & 66,1 \\
Tecnologías productivas & 6 & 3,64 & 159 & 96,36 \\
Tecnología de información y & & & & \\
comunicaciones & 5 & 3,03 & 160 & 96,97 \\
Envases o etiquetado & 5 & 3,03 & 160 & 96,97 \\
Procesamiento & & & & \\
La explotación cuenta con: & 45 & 27,28 & 120 & 72,72 \\
Buenas Prácticas Agrícolas & 5 & 3,03 & 160 & 96,97 \\
HACCP & 5 & 3,03 & 160 & 96,97 \\
Normas ISO & 4 & 2,42 & 161 & 97,58 \\
Resolución sanitaria & & & & \\
\hline
\end{tabular}

HACCP: Análisis de Peligros y Puntos Críticos de Control ISO: International Organization for Standardization 
(Rodrigues, 2012). La conectividad no es la única prioridad a atender para mejorar el uso de las TIC en la agricultura nacional, sino también la generación de contenidos útiles y atractivos, así como la mejora de las capacidades de los potenciales usuarios (FIA, 2008). En el mismo tema, los productores fueron también consultados respecto al principal medio que utilizaban para comunicarse e informarse, siendo: televisión $(70,9 \%)$, radio $(16,4 \%)$, Internet $(10,3 \%)$ y prensa $(2,4 \%)$. Estos resultados son muy relevantes para plantear una estrategia que dé a conocer a los agricultores los instrumentos públicos de apoyo, o para entregarles información de interés.

Por su parte, la innovación reciente que declaran los encuestados es el cultivo de nuevas especies $(72,7 \%) \mathrm{y}$, de forma secundaria, en el manejo del campo $(64,24 \%)$. Las innovaciones relativas a gestión, uso de TIC y agregación de valor en la post cosecha son realizadas solamente por entre un $14 \%$ y un $3 \%$ de los encuestados. A nivel nacional la tasa de innovación declarada en el sector agrícola es una de las más bajas de la economía, con un 9,5\% de las empresas (Ministerio de Economía, Fomento y Turismo, 2018). En este caso, si bien las cifras son significativamente superiores a las nacionales, consideramos que es posible que exista una confusión entre innovación e incorporación de transferencia tecnológica.

En relación a permisos y certificaciones, se presentaron cuatro opciones a los encuestados: Buenas Prácticas Agrícolas (BPA), Análisis de Peligros y Puntos Críticos de Control (HACCP), normas ISO (International Organization for Standardization) y resolución sanitaria. Un $27,28 \%$ de los productores señaló estar certificado por BPA. Las otras opciones tuvieron una incidencia entre el 2 y el 3\%. En el caso específico de la resolución sanitaria, es necesario aclarar que solamente se requiere cuando al producto primario se le realiza alguna transformación. La minoritaria incidencia de las certificaciones puede deberse a que los productores hortícolas tienen como mercado objetivo el interno, por ahora no tan exigente en ese sentido. En efecto, como señalan Beghin et al. (2015), tras realizar una revisión de la evidencia científica, las certificaciones tienen especial impacto cuando se apunta a cadenas de alto valor agregado.

\section{Mecanismos de gestión predial y de comercialización}

Con el objeto de complementar el perfil técnico-productivo de los encuestados se consultó acerca de los mecanismos utilizados para gestionar sus explotaciones y comercializar. Para ello se les preguntó acerca de la formalización de su actividad, el uso de registros, el acceso a financiamiento, el apoyo asociativo y la participación en programas públicos.

Un $87,3 \%$ de los encuestados declara que su explotación tiene inicio de actividades en el Servicio de Impuestos Internos. El promedio nacional para el sector primario es, sin embargo, del 26,2\% (Ministerio de Economía, 2015). No obstante, consideramos que pueden existir inconsistencias en las respuestas de los encuestados a esta sección; dado que, por ejemplo, un $27,7 \%$ no supo concretar cuál era el tipo de personalidad jurídica bajo el que se constituía su empresa. En cuanto al régimen de tributación, un $69,7 \%$ del total de productores declara que impone mediante renta presunta, la cual exime de llevar contabilidad. De hecho, un $71,5 \%$ de los productores señalaba no tener registros económicos de su actividad. Por otro lado, un $80,6 \%$ de los encuestados afirmaba no pertenecer a ningún tipo de asociación técnicoproductiva.

Respecto al financiamiento para las inversiones y gastos operativos prediales, el $49,7 \%$ de los encuestados ha recibido subsidios y el $81,8 \%$ crédito en los últimos 5 años. De los receptores de crédito, el 85\% recurrió al Instituto de Desarrollo Agropecuario (INDAP) como fuente. La banca privada tuvo, sin embargo, una participación casi nula (1 caso). De hecho, el nivel de bancarización de los productores es limitado: sólo un $40 \%$ de los encuestados declaraba utilizar cuenta para administrar los flujos monetarios de la explotación. Para el caso de Chile, los impactos del acceso a crédito son heterogéneos, dependiendo del rubro e incluso del capital social del productor (von Cramon-Taubadel y Saldías, 2014; Reyes y Lensink, 2011).

El 53,3\% de los encuestados declaró haber sido beneficiario de programas públicos en los últimos 5 años. De ellos, el 87,5\% del Programa de Asesoría Técnica (SAT), del Instituto de Desarrollo Agropecuario (INDAP). Los años que cada productor ha sido en promedio beneficiario del programa señalado son 4,18. El Programa de Desarrollo Local (PRODESAL), también de INDAP, fue mencionado sólo por un 9\% de los productores. Estos resultados llaman la atención ya que PRODESAL es el programa público de fomento agrícola más importante en Chile en términos de cobertura y número de beneficiarios. No obstante, puede deberse a que PRODESAL se dirige preferentemente a los agricultores de más reducido tamaño, denominados por INDAP como "multiactivos". Sin embargo, entre los encuestados priman aquellos que, si bien son pequeños agricultores, no son tan reducidos, lo que INDAP califica como perfil "comercial". El Programa SAT está 
dirigido de hecho específicamente a los segundos (Rimisp y Demoscópica, 2017). Sólo un 3\% de los encuestados declaró haber recibido apoyo del sector privado en los últimos 5 años.

En relación a la comercialización, los intermediarios y mayoristas son el principal canal de venta para un $66,7 \%$ de los encuestados. De manera secundaria aparece la venta directa en feria $(15,75 \%)$. La exportación y la agroindustria son minoritarios $(8,4 \%$ y $4,2 \%$, respectivamente), y la venta a supermercados es inexistente. Estos resultados son coherentes con la realidad nacional. La cadena productor-intermediario-centrales de abastecimiento-ferias libres es el principal canal para la pequeña agricultura chilena, sobre todo hortofrutícola (Rivas, 2012).

\section{Valoración acerca de su actividad}

Por último, se presentó a los encuestados una serie de afirmaciones referentes a su actividad como productores hortícolas para que pudieran valorar su grado de acuerdo con ellas. Los principales resultados se presentan en la Tabla 3.

En primer lugar, destaca la confianza que tienen los horticultores en los atributos positivos de sus productos, así como del proceso tras su obtención, lo que se evidencia en la alta valoración de las afirmaciones: "Considero que

Tabla 3. Valoración de afirmaciones relativas a actitudes de los encuestados frente a su actividad hortícola (Escala Likert de 1 a 5)

Table 3. Assessment of statements related to attitudes of the respondents to their horticultural activity (Likert scale from 1 to 5 )

\begin{tabular}{|c|c|c|}
\hline Afirmación & Media & D.E. \\
\hline 1. Necesito mayor inversión para hacer crecer mi producción & 4,55 & 1,12 \\
\hline 2. Considero que mis técnicas de riego son adecuadas & 4,28 & 1,30 \\
\hline 3. Considero que mis técnicas de fertilización de suelo son adecuadas & 4,41 & 1,21 \\
\hline 4. Considero que mis técnicas de propagación de material vegetal son adecuadas & 4,16 & 1,27 \\
\hline 5. Considero que mis técnicas de control de malezas son adecuadas & 4,02 & 1,34 \\
\hline 6. Considero que mis técnicas de control de plagas y enfermedades son adecuadas & 3,97 & 1,29 \\
\hline 7. Considero que mis técnicas de manejo de residuos son adecuadas & 4,26 & 1,07 \\
\hline 8. Considero que mis hortalizas son de buena calidad & 4,76 & 0,62 \\
\hline 9. Considero que mis hortalizas son inocuas & 4,76 & 0,55 \\
\hline 10. Estoy dispuesto a pagar por asesorías especializadas sobre técnicas de producción & 3,54 & 1,72 \\
\hline 11. La venta de mis hortalizas se realiza en un momento oportuno & 4,64 & 0,73 \\
\hline 12. La forma de pago por la venta de mis productos es de mi agrado & 4,34 & 1,30 \\
\hline 13. El precio de venta de mis hortalizas es bueno & 2,66 & 1,74 \\
\hline 14. Estoy dispuesto a pagar por asesorías especializadas sobre comercialización & 3,28 & 1,73 \\
\hline 15. Considero que la formalización es importante para el éxito de mi actividad & 4,57 & 1,03 \\
\hline 16. Considero que los trámites para formalizarse son sencillos & 4,02 & 1,42 \\
\hline 17. Considero que los trámites para obtener certificaciones son sencillos & 2,41 & 1,79 \\
\hline 18. Estoy dispuesto a pagar por asesorías especializadas sobre gestión & 2,59 & 1,83 \\
\hline 19. Tengo una visión positiva sobre el futuro de mi actividad hortícola & 2,47 & 1,74 \\
\hline 20. La introducción de innovaciones en mi sistema productivo ha sido beneficiosa & 3,79 & 1,69 \\
\hline 21. La introducción de innovaciones en mi sistema comercial ha sido beneficiosa & 2,33 & 1,54 \\
\hline 22. La introducción de innovaciones en mi sistema de gestión ha sido beneficiosa & 3,15 & 1,91 \\
\hline $\begin{array}{l}\text { 23. Considero que los ingresos que genera mi actividad permiten que mi grupo familiar } \\
\text { tenga acceso a una buena calidad de vida }\end{array}$ & 4,45 & 1,10 \\
\hline 24. Considero que la cantidad de tiempo que trabajo en mi predio hortícola es adecuada & 3,88 & 1,44 \\
\hline 25. Considero que mi actividad es amigable con el medio ambiente & 4,71 & 0,67 \\
\hline 26. Los instrumentos de apoyo disponibles para el sector hortícola son suficientes & 2,44 & 1,70 \\
\hline 27. Los instrumentos públicos de apoyo que he recibido han sido útiles para mi actividad & 4,32 & 1,39 \\
\hline 28. Los instrumentos privados de apoyo que he recibido han sido útiles para mi actividad & 1,87 & 1,55 \\
\hline 29. Considero que la información disponible sobre instrumentos de apoyo es suficiente & 2,51 & 1,76 \\
\hline 30. La postulación a instrumentos de apoyo para el sector hortícola es sencilla & 3,63 & 1,65 \\
\hline $\begin{array}{l}\text { 31. Considero que la existencia de asociaciones técnico-productivas o comerciales son } \\
\text { beneficiosas para quienes las conforman }\end{array}$ & 3,70 & 1,68 \\
\hline
\end{tabular}


mis hortalizas son de buena calidad" (Promedio $4,76)$, "Considero que mis hortalizas son inocuas" $(4,76)$, "Considero que mi actividad es amigable con el medioambiente" $(4,71)$. Así mismo, en lo relativo a las técnicas productivas utilizadas, por lo general existe acuerdo acerca de su conveniencia, siendo valoradas en este orden: fertilización de suelo $(4,41)$; riego $(4,28)$; manejo de residuos $(4,26)$; propagación de material vegetal $(4,16)$; control de malezas $(4,02)$; y control de plagas y enfermedades $(3,97)$. Así mismo, existe satisfacción con el proceso de venta de las hortalizas: "La venta de mis hortalizas se realiza en un momento oportuno" $(4,64)$ y "La forma de pago por la venta de mis productos es de mi agrado" $(4,34)$; excepto en lo relativo al precio obtenido: "El precio de venta de mis hortalizas es bueno" $(2,66)$. Por otra parte, existe un alto grado de acuerdo en la importancia de formalizar la actividad e incluso en la facilidad de poder hacerlo: "Considero que la formalización es importante para el éxito de mi actividad" $(4,57)$ y "Considero que los trámites para formalizarse son sencillos" $(4,02)$.

A pesar de que en los hechos no se manifiesta con tanta claridad, la innovación productiva y la pertenencia a asociaciones también se perciben como factores de éxito: "La introducción de innovaciones en mi sistema productivo ha sido beneficiosa" $(3,79)$ y "Considero que la existencia de asociaciones técnico-productivas o comerciales son beneficiosas para quienes las conforman" $(3,70)$. Sin embargo, el acuerdo es notablemente menor cuando se evalúan las innovaciones no tecnológicas: "La introducción de innovaciones en mi sistema de gestión ha sido beneficiosa" $(3,15)$ y "La introducción de innovaciones en mi sistema comercial ha sido beneficiosa" $(2,33)$; lo que puede deberse a la falta de familiaridad de los encuestados con ellas. Respecto al apoyo del sector público, hay acuerdo en su impacto positivo: "Los instrumentos públicos de apoyo que he recibido han sido útiles para mi actividad" $(4,32)$. No obstante, se considera la magnitud de dichos apoyos y la información disponible inadecuada: "Los instrumentos de apoyo disponibles para el sector hortícola son suficientes" $(2,51)$ y "Considero que la información disponible sobre instrumentos de apoyo es suficiente" $(2,44)$.

Respecto a la visión general acerca de su actividad, los resultados sugieren un cierto pesimismo en relación a su porvenir: "Tengo una visión positiva sobre el futuro de mi actividad hortícola" $(2,74)$. Un punto crítico para poder crecer es la disponibilidad de recursos financieros: “Necesito mayor inversión para hacer crecer mi producción" $(4,55)$. Pese a la incertidumbre sobre el futuro, existe un amplio acuerdo respecto a la contribución actual que los ingresos generados por la horticultura significan para el hogar: "Considero que los ingresos que genera mi actividad permiten que mi grupo familiar tenga acceso a una buena calidad de vida" $(4,45)$. El nivel de valoración disminuye comparativamente - aunque sigue siendo alto - en lo que respecta al tiempo para obtener dicho ingreso: "Considero que la cantidad de tiempo que trabajo en mi predio hortícola es adecuada" $(3,88)$.

Por último, respecto a la valoración de un posible pago por asesorías especializadas, existe indecisión en el caso de la producción y la comercialización (3,54 y 3,28, respectivamente), así como aversión para los aspectos relacionados con la gestión $(2,59)$. En el caso de las asesorías técnico-productivas, lo anterior puede deberse a que, principalmente en el caso de los pequeños productores, éstos acostumbran a recibirlas dentro de programas de entidades públicas, sobre todo de INDAP. Para las asesorías en gestión y comercialización, es probable que exista desconocimiento de sus potenciales beneficios.

Aunque el análisis de actitudes a partir de la valoración de enunciados es mucho más frecuente en la literatura sobre estudios del consumidor, se ha aplicado previamente para identificar las percepciones de los agricultores incluso en el caso específico de Chile (ej. Mora et al., 2013; Boza et al., 2016). En términos generales, estas investigaciones muestran que, entre pequeños agricultores, existen distintas maneras de percibir su actividad, sobre todo en relación a la actitud frente al cambio. Lo anterior a su vez está condicionado por las características propias de cada productor, como por ejemplo la edad, el nivel educacional, el género o el nivel tecnológico.

\section{CONCLUSIONES}

El presente artículo permite concluir acerca de algunas características generales del grupo bajo estudio, que si bien no pueden ser consideradas representativas de la población total (pequeños horticultores de la Región de O'Higgins), conllevan una aproximación exploratoria. En primer lugar, la mayor parte de los agricultores $(59,39 \%)$ tiene una edad superior a los 50 años y un grupo muy minoritario $(13,94 \%)$ por debajo de los cuarenta. Otro resultado destacable, es que más de la mitad de los productores $(53,34 \%)$ ha cursado sólo educación básica.

Respecto al grupo familiar, el promedio de integrantes es de 3,25. En la mayor parte de los casos $(60,6 \%)$ ninguno de ellos es un menor. Para un $36,9 \%$ de los productores otro miembro del grupo familiar trabaja en el predio. Un $94,5 \%$ de los encuestados tiene acceso a movilización 
particular, un $98,2 \%$ a agua potable, electricidad y establecimientos educacionales, así como un $96,4 \%$ a servicios de salud. Las principales limitaciones están en el acceso a Internet, con un $24,2 \%$ y en la disponibilidad de establecimientos de educación superior en el territorio.

En relación a los sistemas productivos, la superficie utilizada por los encuestados para el cultivo de hortalizas es en promedio 7,8 hectáreas. No obstante, la dispersión de los datos es notoria; de hecho, un $41,2 \%$ de los agricultores está por debajo de las cinco hectáreas. Los productores son principalmente arrendatarios $(69,1 \%)$ o dueños $(26,6 \%)$ de sus predios. Los cultivos más frecuentes entre los encuestados son papa, cebolla, ajo, melón y tomate. La mayor parte de los agricultores $(81,21 \%)$ no tiene ningún trabajador permanente. El 98,2\% de los encuestados usa técnicas convencionales y el $95,2 \%$ cultiva al aire libre; la hidroponía, el cultivo orgánico y el uso de invernaderos son muy poco frecuentes. Un $27,8 \%$ tiene certificación de BPA. Los principales canales de comercialización son intermediarios y mayoristas $(66,7 \%)$.

Respecto a las TIC, el 30,3\% de los productores declara que usa computador, en contraste con la telefonía celular, con el 96,36\%. Las innovaciones, en los casos que los agricultores señalan que existen, se centran en aspectos técnicoproductivos: cultivo de nuevas especies $(72,7 \%)$ y manejo del campo $(64,24 \%)$. La participación en programas públicos se fundamenta en la vinculación a INDAP, a través principalmente del programa SAT $(87,5 \%)$ y de la obtención de crédito (69,5\%). La mayor parte de los encuestados $(71,5 \%)$ no mantiene registros económicos de su actividad y una minoría $(19,4 \%)$ participa de algún tipo de asociación.

Respecto a sus actitudes, destaca la alta valoración (mayor que 4,0) de las afirmaciones relativas a la calidad de sus productos y las técnicas puestas en práctica para su obtención. Así mismo, existe una alta valoración del proceso de venta de las hortalizas, excepto en lo relativo al precio, y de la importancia de formalizar la actividad e incluso de la facilidad de poder hacerlo. Por el contrario, las afirmaciones con poca valoración (menor que 3.0) son relativas a la importancia de innovar en aspectos de gestión y comercialización, a la magnitud e información respecto a instrumentos de apoyo público, así como a una visión positiva del futuro de su actividad.

\section{RECONOCIMIENTOS}

Esta investigación fue desarrollada dentro del proyecto "Centro de Extensión Hortícola" financiado por el concurso de INNOVA-CORFO
"Centro de Extensionismo Tecnológico", línea Alimentos Saludables. Los autores agradecen asimismo a los profesionales que colaboraron en el levantamiento de la información.

\section{LITERATURA CITADA}

Banco Mundial. 2018. Estadísticas crecimiento de la población urbana. Disponible en: https:// datos.bancomundial.org/indicador/SP.URB. GROW (Consulta 25 abril 2018).

Beghin, J., M. Maertens, and J. Swinnen. 2015. Nontariff measures and standards in trade and global value chains. Bioeconomics Working Paper Series No. 2015/02. Department of Earth and Environmental Sciences, University of Leuven, Leuven, Belgium.

Berdegué, J., y F. Rojas. 2014. La agricultura familiar en Chile. Rimisp Grupo de Trabajo Desarrollo con Cohesión Territorial. Documento de Trabajo $N^{\circ}$ 152. 42 p. Rimisp, Santiago, Chile.

Boza, S., M. Cortés, y F. Guzmán. 2015. Caracterización de pequeños empresarios agrícolas beneficiarios de programas de desarrollo local en la Región Metropolitana, Chile. Revista IDESIA 33:135-142. doi: 10.4067/S0718-34292015000100014.

Boza, S., M. Cortés, y T. Muñoz. 2016. Estrategias de desarrollo rural con enfoque de género en Chile: el caso del programa Formación y Capacitación para Mujeres Campesinas. Revista Civilizar 16:63-75.

Bravo, J., y S. Urzúa. 2018. Inmigrantes: Empleo, capital humano y crecimiento. Documento de Trabajo $N^{\circ}$ 47. 31 p. Centro CLAPES UC, Santiago, Chile.

Castro, A. 2012. Familias rurales y sus procesos de transformación: Estudio de casos en un escenario de ruralidad en tensión. Psicoperspectivas 11:180-203. doi: 10.5027/ psicoperspectivas-Vol11-Issue1-fulltext-172.

Cerda, R., D. Egaña, P. Galvez, y D. Masferrer. 2016. Marco conceptual sobre los factores condicionantes de los ambientes alimentarios en Chile. 83 p. Universidad de Chile y Ministerio de Salud, Santiago, Chile.

Echeverría, R., V. Moreira, J. Barrena, and M. Gopinath. 2012. A characterization of Chilean farmers based on their market-production orientation. Ciencia e Investigación Agraria 39:255-264. doi: 10.7764/rcia.v39i2.58.

Eguillor, P. 2017. Agricultura orgánica: agosto de 2017. 24 p. ODEPA, Santiago, Chile. Disponible en https://www.odepa.gob.cl/wpcontent/uploads/2017/12/organico2017.pdf (Consulta 19 abril 2018). 
Espacio y Fomento. 2016. Estudio para el desarrollo de los mercados locales para mejorar el acceso a frutas y hortalizas frescas en las regiones Metropolitana y del General Libertador Bernardo O'Higgins. 46 p. ODEPA, Santiago, Chile.

Etikan, I., S.A. Musa, and R.S. Alkassim. 2016. Comparison of convenience sampling and purposive sampling. American Journal of Theoretical and Applied Statistics 5:1-4.

FAO. 2011. The state of food and agriculture. Women in agriculture: Closing the gender gap for development. 147 p. FAO, Rome, Italy.

FIA. 2008. Tecnologías de Información y Comunicación (TICs) aplicadas en el mundo rural. 42 p. Fundación para la Innovación Agraria, Santiago, Chile.

Garner, E., and Campos, A.P. de la O. 2014. Identifying the "family farming": an informal discussion of the concepts and definitions. ESA-FAO Working Paper No. 14-10. FAO, Rome, Italy.

Gobierno Regional de O'Higgins. 2012. Estrategia regional de innovación 2012-2015. Disponible en https://www.dellibertador. cl/planificacion/estrategia_innovacion_ ohiggins_2012-2015.pdf (Consulta 14 mayo 2018).

Graeub, B., M.J. Chapell, H. Wittman, S. Ledermann, R. Bezner, and B. GemmillHerren. 2016. The state of family farms in the world. World Development 87:1-15. doi: 10.1016/j.worlddev.2015.05.012

INE. 2016. Estadísticas Agrícolas. Informe de Cultivo de Hortalizas. Disponible en http:// historico.ine.cl/canales/chile_estadistico/ estadisticas_agropecuarias/estadisticas_ agricolas/agricolas.php (Consulta 15 mayo 2018).

Maloney, W.F. 2004. Informality revisited. World Development 32:1159-1178. doi: 10.1016/j. worlddev.2004.01.008.

Ministerio de Economía, Fomento y Turismo. 2015. Informe de resultados: Formalidad de los emprendimientos en Chile. Cuarta encuesta de microemprendimiento 2015. Disponible en www.economia.gob.cl/wp-content/ uploads/2016/07/Informe-de-resultadosFormalidad-de-los-emprendimientos-enChile.pdf (Consulta 12 enero 2017).

Ministerio de Economía, Fomento y Turismo. 2018. Principales Resultados Décima Encuesta Nacional de Innovación. Disponible en http://www.economia.gob.cl/wp-content/ uploads/2018/02/Minuta_Innovaci_n_a_o_ referencia_2016.pdf (Consulta 17 abril 2018).
Ministerio de Salud. 2017a. Encuesta Nacional de Salud ENS Chile 2016-2017: Primeros resultados. Disponible en http://www. minsal.cl/wp-content/uploads/2017/11/ENS2016-17_PRIMEROS-RESULTADOS.pdf (Consulta 26 marzo 2018).

Ministerio de Salud. 2017b. Política Nacional de Alimentación y Nutrición. Disponible en http:// www.bibliotecaminsal.cl/wp/wp-content/ uploads/2018/01/BVS_POL\%C3\%8DTICAD E - A L I M E N T A C I \% C $3 \% 93$ N - Y NUTRICI\%C3\%93N.pdf (Consulta 7 mayo 2018).

Mora M., M. Cortés, C. Sanhueza, and C. Sáenz. 2013. Innovation requirements for the development of cactus pear for export: A new item to be incorporated to the Chilean fruit export sector. Acta Horticulturae 995:229-236 doi: 10.17660/ActaHortic.2013.995.27.

Namdar-Irani, M., y J. Aracena. 2014. Mujer agrícola y políticas públicas en Chile. 101 p. Qualitas Consultores, Santiago, Chile.

NEPAD. 2013. Agriculture in Africa: Transformation and outlook. 76 p. New Partnership for African Development, Johannesburg, South Africa.

Observatorio Feria Libre. 2013. Características Económicas y Sociales de Ferias Libres de Chile. FAO, ODEPA y ASOF, Santiago, Chile.

ODEPA. 2018. Estadísticas comercio exterior. Disponible en https://www.odepa.gob.cl/ estadisticas-del-sector/comercio-exterior (Consulta 9 mayo 2018).

Qualitas. 2010. Estudio de caracterización de la pequeña agricultura a partir del VII Censo Nacional Agropecuario y Forestal. Qualitas Consultores e INDAP, Santiago, Chile.

Reyes, A., and R. Lensink. 2011. The credit constraints ofmarket-oriented farmersinChile. Journal of Development Studies 47:18511868. doi: 10.1080/00220388.2011.579111.

Rimisp y Demoscópica. 2017. Línea base de los usuarios de INDAP 2015. INDAP, Rimisp y Demoscópica, Santiago, Chile.

Rios, S., y G. Torres. 2014. El sector agropecuario en la Región de Los Lagos y el paradigma "Chile potencia alimentaria": desafíos para la política agraria nacional. Mundo Agrario 15.

Rivas, T. 2012. Comercialización de productos hortofrutícolas en la pequeña agricultura. 9 p. ODEPA, Santiago, Chile.

Rodrigues, M. 2012. Las TIC en la agricultura latinoamericana: Acceso, usos y políticas. En Seminario "El Uso de las TIC en la agricultura". 11 de diciembre. CEPAL, La Paz, Bolivia. 
Santesmases, M. 2009. DYANE versión 4: Diseño y análisis de encuestas en investigación social y de mercados. 560 p. Ediciones Pirámide, Madrid, España.

Tejada, H. 2014. Venta directa en ferias locales: el proceso de modernización de las ferias libres en Chile. p. 93-96. En O. Sotomayor, M. Rodrigues, y A. Rodríguez (coord.) Agricultura familiar y circuitos cortos: Nuevos esquemas de producción, comercialización y nutrición. CEPAL, Santiago, Chile.

Universidad de Chile. 2014. Encuesta Nacional de Consumo Alimentario. Disponible en http:// web.minsal.cl/sites/default/files/ENCAINFORME_FINAL.pdf. (Consulta 13 enero 2017).

Valdés, X., y L. Rebolledo. 2015. Géneros, generaciones y lugares: cambios en el medio rural de Chile Central. Polis (Santiago) 14:491513. doi: 10.4067/S0718-65682015000300022
Van Vliet, J., A. Schut, P. Reidsma, K. Descheemaeker, M. Slingerland, G. Van de Ven, et al. 2015. De-mystifying family farming: features, diversity and trends across the globe. Global Food Security 5:11-18. doi: 10.1016/j.gfs.2015.03.001

Vera, M.B., y V. Moreira. 2009. Caracterización de la microempresa agrícola del Sur de Chile. IDESIA 27:89-99. doi: 10.4067/S071834292009000300011.

Von Cramon-Taubadel, S., and R. Saldías. 2014. Access to credit and determinants of technical inefficiency of specialized smallholder farmers in Chile. Chilean Journal of Agricultural Research 74:413-420 doi: 10.4067/S0718-58392014000400006

Wichelns, D. 2015. Achieving water and food security in 2050: Outlook, policies, and investments. Agriculture 5:188-220 doi: 10.3390/agriculture5020188 